\title{
Biphasic activation of extracellular signal-regulated kinase (ERK) $1 / 2$ in epidermal growth factor (EGF)-stimulated SW480 colorectal cancer cells
}

\author{
Donghyun Joo ${ }^{1}$, Jong Soo Woo ${ }^{1}$, Kwang-Hyun $\mathrm{Cho}^{2}$, Seung Hyun Han ${ }^{3}$, Tae Sun Min ${ }^{4}$, \\ Deok-Chun Yang ${ }^{5}$ \& Cheol-Heui Yun ${ }^{1, *}$ \\ ${ }^{1}$ Department of Agricultural Biotechnology and Center for Agricultural Biomaterials; Research Institute for Agriculture and Life Sciences, \\ Seoul National University, Seoul 08826, ${ }^{2}$ Department of Bio and Brain Engineering, Korea Advanced Institute of Science and Technology \\ (KAIST), Daejeon 34141, ${ }^{3}$ Department of Oral Microbiology and Immunology, Dental Research Institute, and BK21 Program, School of \\ Dentistry, Seoul National University, Seoul 08826, ${ }^{4}$ National Research Foundation of Korea, Daejeon 34113, ${ }^{5}$ Korean Ginseng Center and \\ Ginseng Genetic Resource Bank, Kyung Hee University, Yongin 17104, Korea
}

Cancer cells have different characteristics due to the genetic differences where these unique features may strongly influence the effectiveness of therapeutic interventions. Here, we show that the spontaneous reactivation of extracellular signalregulated kinase (ERK), distinct from conventional ERK activation, represents a potent mechanism for cancer cell survival. We studied ERK1/2 activation in vitro in SW480 colorectal cancer cells. Although ERK signaling tends to be transiently activated, we observed the delayed reactivation of ERK1/2 in epidermal growth factor (EGF)-stimulated SW480 cells. This effect was observed even after EGF withdrawal. While phosphorylated ERK1/2 translocated into the nucleus following its primary activation, it remained in the cytoplasm during late-phase activation. The inhibition of primary ERK1/2 activation or protein trafficking, blocked reactivation and concurrently increased caspase 3 activity. Our results suggest that the biphasic activation of ERK1/2 plays a role in cancer cell survival; thus, regulation of ERK1/2 activation may improve the efficacy of cancer therapies that target ERK signaling. [BMB Reports 2016; 49(4): 220-225]

\section{INTRODUCTION}

Extracellular signal-regulated kinase (ERK) participates in the regulation of pivotal cell processes, including proliferation,

${ }^{*}$ Corresponding author. Tel: +82-2-880-4802; Fax: +82-2-8864805; E-mail: cyun@snu.ac.kr

http://dx.doi.org/10.5483/BMBRep.2016.49.4.004

Received 9 January 2016, Revised 1 February 2016, Accepted 11 February 2016

Keywords: Biphasic activation, Cancer cell survival, ERK pathway, pERK translocation, SW480 cells differentiation, survival, and apoptosis. It is well established that when epidermal growth factor (EGF) binds to its cognate receptor, Ras recruits Raf to the inner surface of the plasma membrane. Then, phosphorylated Raf stimulates MEK1/2 to phosphorylate ERK1/2 within a conserved Thr-Glu-Tyr (TEY) motif in its activation loop. Phosphorylated ERK1/2 (pERK1/2) subsequently translocates to the nucleus, where it modulates the activity of transcription factors such as Elk-1, Fos, and Myc leading to the induction of a range of cellular responses (1).

Recent findings suggest that monomeric pERK $1 / 2$ and ERK1/2 dimers in the cytoplasm play significant roles in cell signaling. A nuclear translocation sequence (Ser-Pro-Ser, SPS) in ERK2 was identified as a general nuclear translocation signal (2) and this sequence is concealed by binding with scaffold proteins such as KSR1, IQGAP, and PAXIL, which results in the cytoplasmic retention of ERK2. However, the mechanisms underpinning these processes and the cytoplasmic functions of pERK1/2 remain to be elucidated.

Although the ERK pathway is transiently activated in many cells, researchers have demonstrated the spontaneous reactivation of ERK signaling in periodontal ligament (PDL) cells (3) and the osteoblastic cell line, MG-63 (4). Biomolecular approaches suggest that fibroblast growth factor (FGF) stimulation leads to the reactivation of ERK signaling through reactivation of Ras (5) and stimulation with thrombin induced biphasic activation of ERK in vascular smooth muscle (VSM) cells as a result of intermediate heparin-binding protein expression (6). However, the details of the mechanism and outcome of this event are unknown.

The high frequency of mutations in ERK signaling intermediates in various cancers reveals the importance of this pathway in proliferation and differentiation. In colorectal cancer, ERK mutations may drive cancer progression (7). More than $50 \%$ of colorectal cancers harbor Ras mutations and $10 \%$ show mutations in B-Raf (8), indicating that continuous ERK activation as a result of mutation, results in uncontrolled 
proliferation and differentiation. These findings confirm the essential role of ERK signaling in the development of colorectal cancer. Therefore, clear understanding of ERK pathway in colorectal cancer is fundamental to establish therapeutic approaches on these diseases.

In this study, we found a link between a unique pattern of ERK activation (characterized by spontaneous and delayed reactivation) and enhanced EGF-stimulated SW480 colorectal cancer cell survival. The delayed reactivation of ERK is dependent on the activities of factors produced during the primary activation phase. We further show that pERK $1 / 2$ influence cell survival during the secondary activation phase in the cytoplasm, indicating that biphasic ERK activation is a potential target for anti-cancer therapies.

\section{RESULTS}

\section{EGF-stimulated SW480 cells display biphasic phosphorylation of ERK1/2}

In SW480 cells, EGF rapidly induced phosphorylation and subsequent nuclear translocation of ERK1/2 (within five min). The levels of pERK $1 / 2$ gradually declined thereafter, returning to near-basal levels within 60 min (Fig. 1A and B). Although it is reported that activation of the ERK pathway is a transient event (9), researchers illustrate that sustained ERK1/2 activation influences cell fate as well. Traverse et al. showed the prolonged activation of ERK by nerve growth factor (NGF) is required for the differentiation of PC12 neuronal cells (10). Therefore, we tested long-term activation of the ERK pathway in EGF-treated SW480 cells. After the initial peak at 5-10 min, the level of pERK $1 / 2$ gradually declined until 100 min after the initial peak, then increased again, between 200 and $400 \mathrm{~min}$ after initial stimulation (Fig. 1C). Suspecting that this phenomenon may have resulted from EGF retained in the culture media, we washed EGF-stimulated cells with PBS five min after stimulation (after the initial peak in ERK activation), then cultured them in EGF and serum-free medium. Again, we observed the reactivation of ERK1/2 at 200-400 min after stimulation, even following the total withdrawal of EGF at five min after stimulation (Fig. 1D), indicating that sustained activation of the ERK pathway by EGF treatment dose not result from EGF retained in culture media.

\section{Phosphorylated ERK1/2 at the secondary activation phase stays in the cytosol, not in the nucleus of EGF-stimulated SW480 cells}

The nuclear translocation of activated cytosolic signaling proteins, including $\mathrm{pERK} 1 / 2$, initiates various cellular responses. Thus, we investigated the distribution of pERK $1 / 2$ during the second activation phase. Within the experimental period, we assessed whether the observed reactivation resulted from an oscillation in ERK1/2 activation prior to the achievement of equilibrium. After stimulation with EGF, phosphorylated ERK $1 / 2$ rapidly entered into the nucleus within $10 \mathrm{~min}$.
However, pERK1/2 at the late activation phase (around 300 min) did not enter into nucleus, but stayed in the cytoplasm (Fig. 2A). Confocal microscopy revealed the presence of pERK1/2 in the nuclei of EGF-stimulated SW480 cells at five min after stimulation. However, It was not detected in the nucleus at the later activation phase, although the overall pERK1/2 levels increased (Fig. 2B).

We next examined whether the retained pERK $1 / 2$ in the cytoplasm at the later activation phase influence the expression of IL-8, an ERK target gene (11). The patterns of IL8 mRNA expression (Fig. 2C) was consistent with the finding that pERK $1 / 2$ does not translocate to the nucleus during the second activation phase.

\section{Blocking primary pERK1/2 activation and protein trafficking prevent biphasic ERK1/2 activation}

Until now, our key findings included the biphasic activation of ERK1/2 in SW480 cells and the differential intracellular localization of $\mathrm{pERK} 1 / 2$ in the two activation phases. The

A

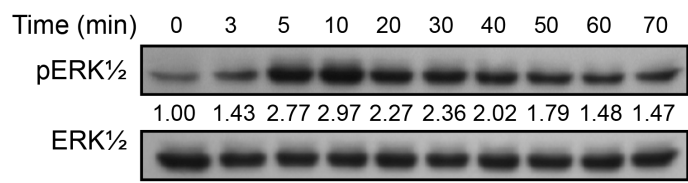

B 5 min after EGF stimulation

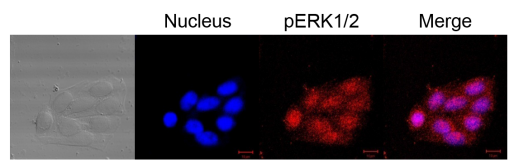

C

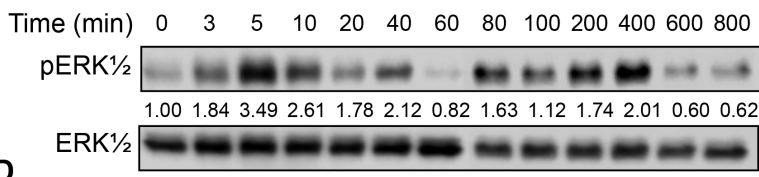

Time (min) $0 \begin{array}{llllllllll}3 & 5 & 10 & 20 & 40 & 60 & 80 & 100 & 200 & 400600800\end{array}$

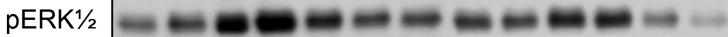
$\begin{array}{lllllllllllll}1.00 & 1.51 & 2.42 & 2.68 & 2.02 & 1.48 & 1.17 & 1.58 & 1.65 & 2.25 & 1.92 & 0.87 & 0.30\end{array}$

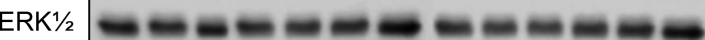

Fig. 1. Patterns of ERK activation in EGF-stimulated SW480 cells. (A) SW480 cells were treated with EGF $(10 \mathrm{ng} / \mathrm{ml})$ for the indicated time periods and the levels of ERK $1 / 2$ and pERK1/2 were measured by Western blot analysis. (B) SW480 cells treated with $10 \mathrm{ng} / \mathrm{ml}$ EGF for five min were labeled with anti-pERK1/2 antibodies (red) and DAPI (blue). Then, the localization of pERK1/2 was monitored by confocal microscopy. Scale bar, $10 \mu \mathrm{m}$. (C, D) Following stimulation with EGF for five min, either EGF stimulation continued $(\mathrm{C})$ or the EGF-containing medium was replaced with serum-free medium (D). Protein extracts were immunoblotted with anti-pERK1/2 and anti-ERK1/2 antibodies. 
A

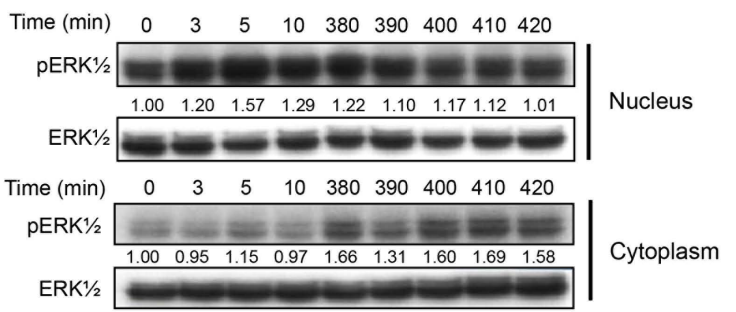

B

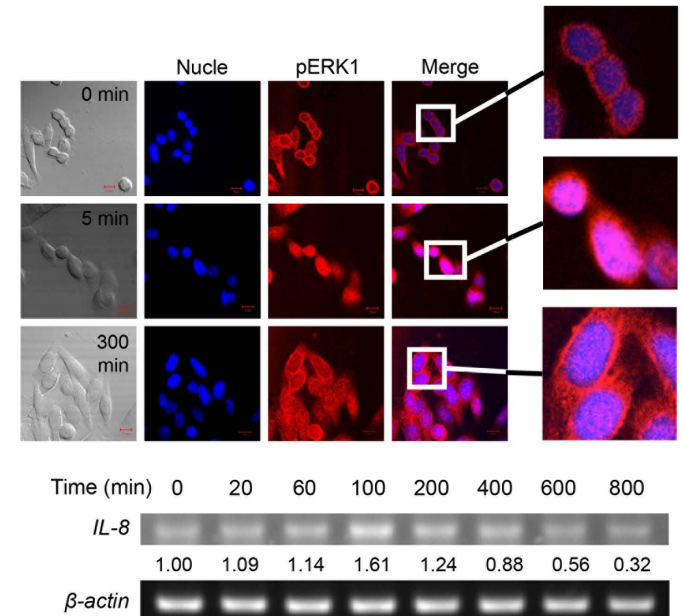

Fig. 2. Localization of $p E R K 1 / 2$ during the second activation phase and its effects on IL-8 expression. (A) SW480 cells were stimulated with EGF $(10 \mathrm{ng} / \mathrm{ml})$ for the indicated periods of time, then split into nuclear and cytosolic fractions using Nuclear and Cytoplasmic Extraction Reagents as described in the Materials and Methods section. The extracts were then immunoblotted with anti-pERK $1 / 2$ and anti-ERK1/2 antibodies. (B) SW480 cells were stimulated with EGF $(10 \mathrm{ng} / \mathrm{ml})$ for the indicated periods. pERK1/2 was detected using anti-phospho ERK $1 / 2$ antibodies and Alexa Fluor $^{(R)}$ 647-conjugated anti-rabbit IgG. Cell nuclei were stained with DAPI. (C) SW480 cells were stimulated with EGF $(10 \mathrm{ng} / \mathrm{ml})$ and the induction of IL-8 RNA was analyzed by RT-PCR assay as described in the Materials and Methods section.

resulting data suggest that the early and late responses result from different mechanisms. To distinguish these mechanisms, we pretreated the cells with the ERK inhibitor, U0126. Pretreatment with U0126 for 60 min completely blocked, not only the primary induction of ERK phosphorylation, but also the secondary activation by EGF stimulation (Fig. 3A). And additional stimulation of U0126-pretreated SW480 cells with FBS at $300 \mathrm{~min}$ induced the rapid phosphorylation of ERK $1 / 2$ (Fig. 3B, lane D), implying that ERK signaling is intact in U0126 pretreated cells at $300 \mathrm{~min}$ and the abolishment of later activation does not result from the sustained effects of U0126. This dataset indicates that the first activation is critical for the later ERK activation in EGF stimulated SW480 cells.

Therefore, we hypothesized that intracrine, autocrine, or paracrine signals triggered by the initial activation, is responsible for the subsequent reactivation of ERK signaling.
A

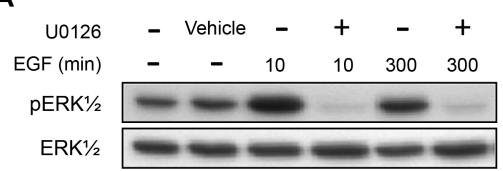

B

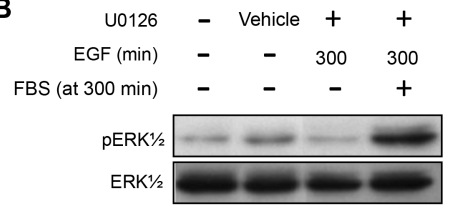

C

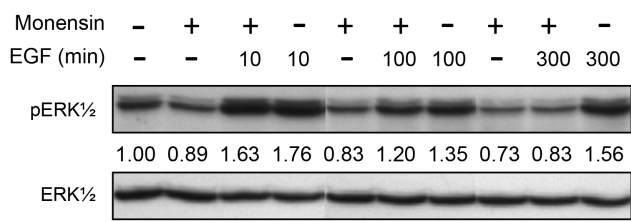

Fig. 3. Activation of ERK signaling following the inhibition of primary ERK activation or protein transport. (A) SW480 cells were incubated with or without U0126 (20 $\mu \mathrm{M})$ for $60 \mathrm{~min}$ and then stimulated with EGF $(10 \mathrm{ng} / \mathrm{ml})$ for 10 or 300 min. (B) SW480 cells, pretreated with or without U0126, were stimulated with EGF for the indicated time periods. *Media was replaced with $20 \%$ FBS at $300 \mathrm{~min}$ and cells were lysed $10 \mathrm{~min}$ later. (C) SW480 cells were pretreated with or without monensin for $60 \mathrm{~min}$ and then stimulated with EGF $(10 \mathrm{ng} / \mathrm{ml})$. Protein extracts were immunoblotted using anti-ERK1/2 and anti-pERK1/2 antibodies.

To test this, we pretreated serum-starved SW480 cells with monensin, a protein transport inhibitor for 60 min prior to EGF stimulation. Although monensin did not block primary ERK activation, reactivation of ERK $1 / 2$ at 300 min did not occur in monensin-pretreated cells and pERK $1 / 2$ levels decreased to basal levels (Fig. 3C). These results suggest that ERK activation at the late phase depends on initial activation, which may generate intracrine, autocrine, or paracrine signals.

\section{Blocking secondary activation enhances apoptosis in SW480 cells}

One hypothesis documented in the literature is that cytoplasmic $\mathrm{pERK} 1 / 2$ activated by MEK2 promotes cancer cell survival (12). Since phosphorylated ERK1/2 during secondary activation also remains in the cytoplasm, we hypothesized that biphasic activation of ERK plays a role in survival of colon cancer cells. First, SW480 cells were starved to minimize the effects of serum on ERK signaling. While this promoted caspase 3 activation, EGF treatment suppressed activation of caspase 3 in SW480 cells. Because the mutated KRAS driven-activation of ERK1/2 in SW480 cells suppressed caspase 3 activation, the inhibition of ERK activity by $U 0126$ treatment increased caspase 3 activity. In contrast, the stimulation with EGF for 30 min resulted in suppression of 
caspase 3 activity, suggesting survival signal activation by EGF stimulation. However, inhibition of the primary ERK activation through pretreatment with U0126 reduced caspase 3 activity by about $10 \%$. When secondary activation was solely inhibited through treatment with U0126 for 60 min prior to sample preparation (treated at $240 \mathrm{~min}$ ), caspase 3 activity significantly increased (Fig. 4A), suggesting that the reactivation of ERK participates in anti-apoptotic signaling. Furthermore, inhibition of both primary and secondary ERK activation significantly increased caspase 3 activity (Fig. 4A), indicating that the spontaneous reactivation of ERK protects cells from serum starvation-induced apoptosis.

Since AKT is a critical signaling pathway to regulate cell survival and death (13) and EGF activates this AKT signaling in SW480 cells, we examined AKT activation in EGF-stimulated SW480 in the early and late activation phases. EGF induced phosphorylation of AKT within 10 min regardless of U0126 pretreatment, however, did not activate AKT signaling at 300 min (Fig. 4B), suggesting that AKT is not responsible for regulation of caspase 3 at the later ERK activation phase.

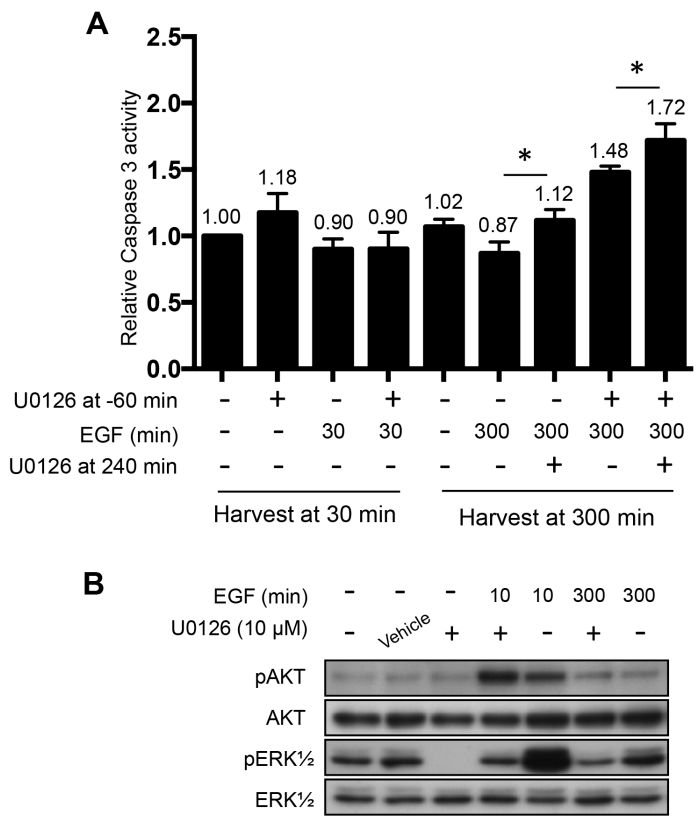

Fig. 4. Caspase 3 activity during the second activation phase and activation of AKT signaling in EGF-stimulated SW480 cells pretreated with U0126. (A) SW480 cells were incubated with or without U0126 $(10 \mu \mathrm{M})$ for $60 \mathrm{~min}$ and then stimulated with EGF (10 ng/ml) for 30 or $300 \mathrm{~min}$. Lanes 7 and 9: samples from cells treated with U0126 $(10 \mu \mathrm{M})$ for $60 \mathrm{~min}$ prior to protein extraction. Protein extracts $(50 \mu \mathrm{g})$ were incubated with Ac-DEVD-AMC at $37^{\circ} \mathrm{C}$ for $30 \mathrm{~min}$. (B) SW480 cells were incubated with or without U0126 $(10 \mu \mathrm{M})$ for $60 \mathrm{~min}$ and then stimulated with EGF (10 $\mathrm{ng} / \mathrm{ml}$ ) for 10 or $300 \mathrm{~min}$. Protein extracts were immunoblotted using anti-ERK1/2, anti-pERK1/2, anti-AKT, and anti-pAKT antibodies. *indicates $\mathrm{P}$ value $\leq 0.05$.

\section{DISCUSSION}

Mutations in ERK signaling components are implicated in development of several cancers. The significance of this pathway in colorectal cancer is well documented; however, data related to specific features, such as biphasic activation, are lacking. Here, we propose biphasic activation of ERK pathway in EGF-stimulated SW480 cells. First, we observed biphasic activation of ERK in SW480 cells upon EGF stimulation and further, investigated whether this phenomenon is a general characteristic in cancer cell lines. However, none of tested cell lines displayed significant levels of pERK $1 / 2$ during the later phases after EGF stimulation (Fig. S1). HeLa and MCF7 cells do not harbor mutations in the ERK pathway, so the ERK activation pattern in these two cell lines represents the general activation pattern of ERK (i.e., rapid activation followed by dephosphorylation). Other cancer cell lines (A549, HCT116, and HT29) harboring K-Ras mutations showed unique patterns of ERK activation indicating that cancer cells exhibit distinct activation profiles. Although HCT116, HT29 and SW480 cells are derived from the same tissue (the colon), it was noting that they showed different activation patterns of ERK signaling. These observations suggest that patterns of EGF-dependent ERK activation vary widely among malignant cell types. Therefore, molecular therapies for specific cancers should consider the cellular context of each tumor cell.

The duration of ERK activation may influence cell fate determination. Indeed, the specific combination of early signals and signals generated by immediate gene products determine the ultimate biological outcome of signaling (14). For example, two distinct activations of ERK pathway between $\mathrm{G} 0$ and $\mathrm{S}$ phase of the cell cycle are required for mitogenesis of cells that are not continuously stimulated (15). In this context, increased initial ERK activation leads to expression of CD44, which functions as a co-receptor, and the induction of CD44 isoform results in sustained ERK activation, which is required for the successful initiation of the G1-S transition. Moreover, other studies have implicated the function of cellular proteins, including CD44v6, MEKs, and, scaffold proteins in the biphasic activation of ERK $(12,16,17)$. Therefore, we tested potential involvement of these molecules in biphasic ERK activation using our experimental setting; however, we detected no changes in EGF-stimulated SW480 cells (Fig. S2).

Although we could not clarify the mechanism details, previous findings suggest potential mediators may be involved. First, protein kinase $\mathrm{C}$ (PKC) may play a role in the late phase activation of ERK signaling. Because respiratory syncytial virus (RSV) induces biphasic activation of ERK through PKC activation, ERK activation in the late stage after EGF treatment may be triggered through PKC signaling pathway (18). Additionally, EGF receptor (EGFR) may regulate this reactivation of ERK signaling since endocytosis of EGFR is 
responsible for sustained ERK activation. However, this process is reported to induce continuous and sustained activation, not reactivation at the later period (19). In addition, biphasic activation is only observed in a particular cancer cell line, not a broad range of cells. Therefore, additional studies are necessary to elucidate the mechanisms underpinning biphasic activation.

Although we observed biphasic ERK activation only in SW480 cells, other evidence supports its occurrence in other cell types. For example, an early study of pERK $1 / 2$ translocation, reported the localized pERK $1 / 2$ in the cytoplasm of $\Delta$ Raf-1:ER cells after the stimulation with serum (20). Platelet-derived growth factor (PDGF) activates PI3K and PKC signaling, which further prolongs the activation of the ERK pathway in Swiss 3T3 cells (21). Cell cycle studies suggest there is a crosstalk between biphasic activation and growth-regulatory signaling. For example, early and late activation of PI3K and PKC by PDGF may be essential for both G1/S progressions $(22,23)$; while two separate pulses of growth factor are necessary to stimulate mitogenesis (15). Sustained pERK1/2 in cytoplasm is reported to regulate cell death by interacting with cytoplasmic substrates, including cytosolic phospholipase A2 (CPLA2) and ribosomal S6 kinase (RSK). Cytosolic pERK1/2 activates CPLA2, which suppresses apoptosis (24). A high level of CPLA2 has been observed in clinical colon tumor biopsies (25), indicating that cytosolic pERK $1 / 2$ in the late activation phase regulates cell death. In addition, the cytoplasmic interactions between pERK $1 / 2$ and RSK play a critical role in cell survival by regulating pro- and anti-apoptotic protein expression (26). It appears that cytoplasmic pERK1/2 generated during the second phase contributes to cell survival by inhibiting caspase 3 activity in SW480 cells. However, further investigation is needed to determine the importance of this potential regulatory mechanism.

Investigators have proposed "oncogene addiction"; where cancers rely on mutations of single signaling intermediates for their survival, therefore, therapy which inhibits the activity of this mutated signaling may be critical to induce apoptosis or regression of those tumors (27). Based on this theory, laboratory studies and clinical trials have been conducted using agents that inhibit oncogenic components of ERK signaling pathways including EGFR, Ras, and Raf $(8,28)$. Therefore, the regulation of ERK signaling and its biphasic activation will provide the evidence to develop a therapeutic strategy for certain types of colorectal cancers.

\section{MATERIALS AND METHODS}

\section{Cell lines and reagents}

Human colorectal carcinoma cells (SW480, CCL-228 ${ }^{\mathrm{TM}}$ ) were purchased from the American Type Culture Collection (ATCC, Manassas, VA) and maintained in RPMI 1640 medium (Invitrogen, Grand Island, NY) supplemented with $10 \%$ fetal bovine serum (FBS) and 1\% antibiotics (Invitrogen). After serum starvation for $24 \mathrm{~h}$, the cells were stimulated with $10 \mathrm{ng} / \mathrm{ml} \mathrm{EGF}$ (Sigma-Aldrich, St. Louis, MO) diluted in serum-free medium. The inhibition of ERK phosphorylation was achieved using U0126 (Calbiochem- Novabiochem, San Diego, CA), a selective MEK1/2 inhibitor. The cells were pretreated with U0126 for 60 min prior to EGF stimulation followed by treatment with GolgiStop $^{\text {TM }}$ (BD Biosciences, San Diego, CA), which contains monensin, in protein trafficking inhibition experiments.

\section{Westem blotting}

Total cellular protein extracts were prepared in RIPA lysis buffer $(150 \mathrm{mM} \mathrm{NaCl}, 1 \% \mathrm{NP}-40,0.5 \%$ deoxycholate, $0.1 \%$ SDS, $50 \mathrm{mM}$ Tris- $\mathrm{HCl}[\mathrm{pH}$ 7.4] with a protease inhibitor cocktail [Roche, Mannheim, Germany]). NE-PER Nuclear and Cytoplasmic Extraction Reagents (Pierce Biotechnology, Rockford, IL) were used to separate the nuclear and cytoplasmic proteins according to the manufacturer's instructions. Target proteins were detected using antibodies specific to ERK1/2 (Chemicon, Temecula, CA), pERK1/2, Akt, and pAkt (Cell Signaling, Danvers, MA). Multi-Gauge software (Fujifilm, Tokyo, Japan) was used to analyze protein signal intensity. The probed membranes were stripped using Restore ${ }^{\mathrm{TM}}$ Western Blot Stripping Buffer (Pierce Biotechnology) and then reprobed.

\section{Confocal microscopy}

The treated cells were fixed with $4 \%$ paraformaldehyde in PBS for $10 \mathrm{~min}$ at $4^{\circ} \mathrm{C}$. They were then permeabilized using $0.5 \%$ Triton X-100 for $45 \mathrm{~min}$ and subsequently incubated with anti-pERK1/2 antibodies (Cell Signaling) for $90 \mathrm{~min}$ at room temperature. The cells were then incubated with anti-rabbit IgG conjugated with Alexa Fluor ${ }^{(R)} 647$ for 60 min and their nuclei stained with 4',6-diamidino-2-phenylindole (DAPI, Invitrogen). pERK1/2 localization was assessed using a confocal microscope (Carl Zeiss AG, Jena, Germany) from sequential $1-\mu \mathrm{m}$ images processed with LSM 510 software (Carl Zeiss AG).

\section{Reverse transcription-polymerase chain reaction (RT-PCR)} Total RNA was isolated from SW480 cells using TRIzol ${ }^{\circledR}$ reagent (Invitrogen). mRNA was reverse-transcribed to complementary DNA (cDNA) using Superscript III RT (Invitrogen). Sense and anti-sense oligonucleotide primers were designed based on sequence information from the NCBI Genome Database. Multi-Gauge software (Fujifilm) was used to analyze the relative intensities.

\section{Detection of caspase 3 activity}

Protein extracts prepared using RIPA lysis buffer without protease or phosphatase inhibitors were transferred to FluoroNunc $^{\mathrm{TM}}$ plates and incubated with the caspase 3 fluorometric substrate Ac-DEVD-AMC (Upstate Biotechnology, Lake Placid, NY) for $30 \mathrm{~min}$ at $37^{\circ} \mathrm{C}$. Fluorescence was measured using a Gemini XPS spectrofluorometer (Molecular Devices, Sunnyvale, CA) (excitation, 360 nm; emission, 460 
nm). The data were analyzed using Prism 6 (GraphPad Software, San Diego CA).

\section{Statistical analysis}

The data are presented as the mean \pm standard deviation (SD). The significance was determined by Student's t-test using Prism 6 software.

\section{ACKNOWLEDGEMENTS}

This work was supported by grants from the Next-Generation BioGreen 21 program (PJ01112401), the Rural Development Administration, and the National Research Foundation of Korea (NRF) funded by the Ministry of Education, Science and Technology (2010-0003291, 2013R1A1A1A05010865, and 2015 M3A9A7067220), Republic of Korea.

\section{REFERENCES}

1. Yang SH, Sharrocks AD and Whitmarsh AJ (2003) Transcriptional regulation by the MAP kinase signaling cascades. Gene 320, 3-21

2. Chuderland D, Konson A and Seger R (2008) Identification and characterization of a general nuclear translocation signal in signaling proteins. Mol Cell 31, 850-861

3. Zeldich E, Koren R, Nemcovsky C and Weinreb M (2007) Enamel matrix derivative stimulates human gingival fibroblast proliferation via ERK. J Dent Res 86, 41-46

4. Huang Z, Cheng SL and Slatopolsky E (2001) Sustained activation of the extracellular signal-regulated kinase pathway is required for extracellular calcium stimulation of human osteoblast proliferation. J Biol Chem 276, 21351-21358

5. Nakayama K, Satoh T, Igari A, Kageyama R and Nishida E (2008) FGF induces oscillations of Hes1 expression and Ras/ERK activation. Curr Biol 18, R332-334

6. Sastre AP, Grossmann S, Reusch HP and Schaefer M (2008) Requirement of an intermediate gene expression for biphasic ERK $1 / 2$ activation in thrombin-stimulated vascular smooth muscle cells. J Biol Chem 283, 25871-25878

7. Sancho E, Batlle E and Clevers H (2004) Signaling pathways in intestinal development and cancer. Annu Rev Cell Dev Biol 20, 695-723

8. Roberts PJ and Der CJ (2007) Targeting the Raf-MEK-ERK mitogen-activated protein kinase cascade for the treatment of cancer. Oncogene 26, 3291-3310

9. Lefloch R, Pouyssegur J and Lenormand P (2008) Single and combined silencing of ERK1 and ERK2 reveals their positive contribution to growth signaling depending on their expression levels. Mol Cell Biol 28, 511-527

10. Traverse $\mathrm{S}$, Gomez N, Paterson $\mathrm{H}$, Marshall $\mathrm{C}$ and Cohen $P$ (1992) Sustained activation of the mitogen-activated protein (MAP) kinase cascade may be required for differentiation of PC12 cells. Comparison of the effects of nerve growth factor and epidermal growth factor. Biochem J 288 (Pt 2), 351-355

11. Sparmann A and Bar-Sagi D (2004) Ras-induced inter- leukin-8 expression plays a critical role in tumor growth and angiogenesis. Cancer Cell 6, 447-458

12. Skarpen E, Flinder LI, Rosseland CM et al (2008) MEK1 and MEK2 regulate distinct functions by sorting ERK2 to different intracellular compartments. FASEB J 22, 466-476

13. Franke TF, Hornik CP, Segev L, Shostak GA and Sugimoto C (2003) PI3K/Akt and apoptosis: size matters. Oncogene 22, 8983-8998

14. Murphy LO, Smith S, Chen RH, Fingar DC and Blenis J (2002) Molecular interpretation of ERK signal duration by immediate early gene products. Nat Cell Biol 4, 556-564

15. Jones SM and Kazlauskas A (2001) Growth-factor-dependent mitogenesis requires two distinct phases of signalling. Nat Cell Biol 3, 165-172

16. Casar B, Pinto A and Crespo P (2008) Essential role of ERK dimers in the activation of cytoplasmic but not nuclear substrates by ERK-scaffold complexes. Mol Cell 31, 708-721

17. Cheng C, Yaffe MB and Sharp PA (2006) A positive feedback loop couples Ras activation and CD44 alternative splicing. Genes Dev 20, 1715-1720

18. Monick M, Staber J, Thomas K and Hunninghake G (2001) Respiratory syncytial virus infection results in activation of multiple protein kinase $\mathrm{C}$ isoforms leading to activation of mitogen-activated protein kinase. J Immunol $166,2681-2687$

19. Sorkin A and von Zastrow M (2009) Endocytosis and signalling: intertwining molecular networks. Nat Rev Mol Cell Biol 10, 609-622

20. Volmat V, Camps M, Arkinstall S, Pouyssegur J and Lenormand P (2001) The nucleus, a site for signal termination by sequestration and inactivation of p42/p44 MAP kinases. J Cell Sci 114, 3433-3443

21. Grammer TC and Blenis J (1997) Evidence for MEKindependent pathways regulating the prolonged activation of the ERK-MAP kinases. Oncogene 14, 1635-1642

22. Balciunaite E, Jones S, Toker A and Kazlauskas A (2000) PDGF initiates two distinct phases of protein kinase $\mathrm{C}$ activity that make unequal contributions to the $\mathrm{GO}$ to $\mathrm{S}$ transition. Curr Biol 10, 261-267

23. Jones SM, Klinghoffer $R$, Prestwich GD, Toker $A$ and Kazlauskas A (1999) PDGF induces an early and a late wave of PI 3-kinase activity, and only the late wave is required for progression through G1. Curr Biol 9, 512-521

24. Nakanishi $M$ and Rosenberg DW (2006) Roles of CPLA2alpha and arachidonic acid in cancer. Biochim Biophys Acta 1761, 1335-1343

25. Soydan AS, Tavares IA, Weech PK, Temblay NM and Bennett A (1996) High molecular weight phospholipase A2 and fatty acids in human colon tumours and associated normal tissue. Eur J Cancer 32A, 1781-1787

26. Ballif BA and Blenis J (2001) Molecular mechanisms mediating mammalian mitogen-activated protein kinase (MAPK) kinase (MEK)-MAPK cell survival signals. Cell Growth Differ 12, 397-408

27. Sharma SV and Settleman J (2007) Oncogene addiction: setting the stage for molecularly targeted cancer therapy. Genes Dev 21, 3214-3231

28. Fang JY and Richardson BC (2005) The MAPK signalling pathways and colorectal cancer. Lancet Oncol 6, 322-327 